\title{
An Ex Vivo Porcine Spleen Perfusion as a Model of Bacterial Sepsis
}

\author{
Wen Y. Chung \#1, Joseph J. Wanford \#2, Rohan Kumar 1, John D. Isherwood 1, Richard D. Haigh 2, \\ Marco R. Oggioni 2, Ashley R. Dennison 1 and Giuseppe Ercoli 2,3 \\ ${ }^{1}$ Department of Hepatobiliary and Pancreatic Surgery, Leicester General Hospital, University of Hospitals of Leicester NHS Trust, Leicester, UK; \\ ${ }^{2}$ Department of Genetics and Genome Biology, University of Leicester, Leicester, UK; ${ }^{3}$ Centre for Inflammation and Tissue Repair, Division of Medicine, \\ University College Medical School, London, UK
}

\begin{abstract}
An ex vivo, porcine spleen perfusion model was established to study the early events occurring in the spleen prior to the onset of bacterial sepsis, using organs retrieved from animals slaughtered for food production. Porcine spleens were harvested from adult pigs and connected to a normothermic extracorporeal perfusion circuit. Constant perfusion of heparinized blood was performed for 6 hours. After injection of Streptococcus pneumoniae to the circuit, serial samples of both blood and spleen biopsies were collected and analyzed. Functionality of the perfused organs was assessed by monitoring the blood-gas parameters, flow rate and filtering capability of the organ. Interestingly, we observed full clearance of bacteria from the blood and an increase in bacterial counts in the spleen. Classical histology and immunohistochemistry on biopsies also confirmed no major damage in the organ architecture and no changes in the immune cell distribution other than the presence of clusters of pneumococci. A time-course study confirmed that each focus of infection derived from the replication of single pneumococcal cells within splenic macrophages. The model proposed - in line with the 3 Rs principles - has utility in the replacement of experimental animals in infection research. Murine models are prevalently used to study pneumococcal infections but are often not predictive for humans due to substantial differences in the immune systems of the two species. This model is designed to overcome these limitations, since porcine immunology, and splenic architecture in particular, closely resemble those of humans.
\end{abstract}

\section{Introduction}

Animal models are still commonly used to study infectious diseases caused by bacterial pathogens. Thousands of animal models have been developed, each specifically designed to study a particular type of infection and its cognate pathology. Rodents are the most commonly used animals due to their low cost and the ease of use but several other animal species have been used as models of bacterial infections (Fink, 2014).

National and international legislation, media and public opinion are encouraging the development of alternative approaches in biomedical research that avoid the use of experimental animals (EU, 2010). New biomedical research funding agencies have emerged in order to guide and support forward-thinking scientists who are putting their efforts towards finding more ethical alternatives to animal experimentation, and many pharmaceutical companies are now also embracing the 3Rs principles (Prescott and Lidster, 2017; Fleetwood et al., 2015).
Beyond the important ethical concerns around the use of experimental animals, one of the main limitations of the use of these models is their lack of translatability to humans (Steiniger, 2015; Mestas and Hughes, 2004; Seok et al., 2013). The majority of animal-based pre-clinical research in the field of bacterial infections, particularly in the industrial setting, aims at the development of new antimicrobial compounds and vaccines. However, some of these animal models do not translate to humans and there are a number of cases in which compounds that showed efficacy in pre-clinical studies later failed in clinical trials (Mak et al., 2014; Mitka, 2011).

In this study, we describe an innovative ex vivo model of infection using local, abattoir-sourced porcine spleens, which allowed the replacement of the use of protected animals. The model has been successfully used in a recent study (Ercoli et al., 2018) and will be extensively described in this article. Autologous, ex vivo perfusion maintains normal physiological parameters, histological characteristics and function for

\footnotetext{
\# contributed equally

Received May 13, 2018; Accepted July 27, 2018; Epub August 3, 2018; () The Authors, 2018.

ALTEX 36(1), 29-38. doi:10.14573/altex.1805131

Correspondence: Giuseppe Ercoli, PhD, Centre for Inflammation and Tissue Repair Division of Medicine, University College of London, London WC1E 6JF, UK (g.ercoli@ucl.ac.uk)
}

This is an Open Access article distributed under the terms of the Creative Commons Attribution 4.0 International license (http//creativecommons. org/licenses/by/4.0/) which permits unrestricted use, distribution and reproduction in any medium, provided the original work is appropriately cited. 
prolonged periods, and allows for a detailed analysis of organ responses to various biochemical and hormonal changes (Imber et al., 2002b,a; Butler et al., 2002; Daniel et al., 2018). Our group has previously developed other ex vivo porcine organ perfusion models to study organ physiology (Chung et al., 2013). The main aim of this model was to study the early stages of bacterial sepsis. Porcine spleen is an ideal choice for this work since pigs have already emerged as relevant biomedical models to study infections (Meurens et al., 2012; Brogaard et al., 2016) and spleen is an organ with a well-defined role in the pathogenesis of invasive bacterial disease (Horan and Colebatch, 1962; Theilacker et al., 2016; Shinefield et al., 1966). Indeed, the spleen actively works as a blood filter, trapping the bacteria and killing them during bacteremia and sepsis. Furthermore, a porcine spleen more closely resembles the human organ than the murine one, with high numbers of circulating neutrophils, comparable microanatomy and subpopulations of splenic and lymph node macrophages, similar T cell subpopulations and cytokine profiles (Hein and Griebel, 2003). Collectively, pigs and humans share more than $80 \%$ of similarity in immune parameters. The majority of human genes that were lost through evolution in the mouse were retained in the pig, with chemokines and both IL-10 and chitinase family members being mostly conserved (Dawson et al., 2013; Dawson, 2011). Spleen perfusion models have already been described both for humans (Buffet et al., 2006) and pigs (Boxall, 1969), but the innovative aspect of this study is the perfusion and infection of organs sourced from the food chain, therefore strongly reducing the need of experimental animals in pre-clinical research. So far, ethical and economic concerns limited the use of pigs to study infectious diseases. With this new ex vivo model we propose to overcome these limitations by providing a new, ethical and cost-effective tool that aims to investigate the dynamics of bacterial infections and to produce results that are more easily and effectively translated to humans.

\section{Animals, materials and methods}

\section{Spleen retrieval}

Spleens were obtained from Large White pigs weighing $45-60 \mathrm{~kg}$ (7 pig spleens were harvested and perfused for this study), which were sourced from Joseph Morris abattoir (South Kilworth, Leicester, UK) following the routine slaughtering procedure. Animals received adequate husbandry, and necessary measures were taken to minimize pain, distress, and suffering during the slaughtering process in accordance with the UK and EU laws (EC, 2009). Pigs were killed by terminal exsanguinations after stunning according to Home Office regulations. At this stage, blood was harvested (1 1) from the jugular vein in non-sterile conditions and 25,000 IU of heparin (LEO Laboratories Limited, UK) were added to prevent coagulation. Intact spleens were retrieved within 5 min post mortem; following median laparotomy and sternotomy, the celiac trunk was accessed to isolate the main splenic artery and vein. Other vessels were clamped and tied, and the spleen was resected en bloc. The en bloc spleen was then transferred to an ice tray where further detailed dissection was carried out. A 10-French catheter was inserted into the splenic artery via the coeliac trunk origin and 11 of Soltran preservation solution (Baxters, UK) containing 5,000 IU of heparin and 10,000 IU of human urokinase (Syner-Medica Ltd, UK) was flushed to remove any residual blood clots. Organs were then transported to the laboratory in bags containing preservation solution on ice.

\section{Ex vivo spleen perfusion}

Ex vivo perfusion was achieved by means of an extracorporeal circuit similar to those used for cardiopulmonary extracorporeal bypass surgery (Medtronic Inc., Minneapolis, MN, USA) (Fig. 1b). The time between the slaughter procedure and the start of perfusion was in the range of 75-90 $\mathrm{min}$. Normothermia was ensured by the use of a heat chamber set at $39^{\circ} \mathrm{C}$ (which allowed

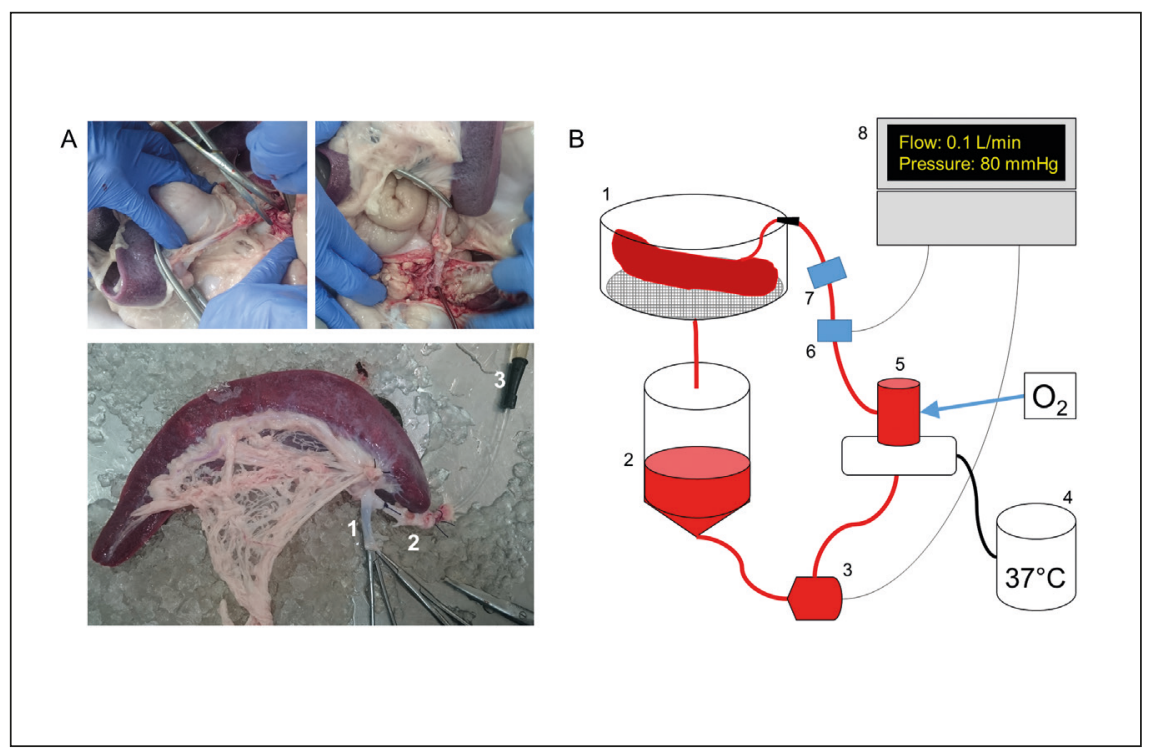

Fig. 1: Optimization of organ retrieval and set-up of the perfusion system The spleen is collected immediately after slaughter from the abattoir. The splenic vein (1) and artery (2) are isolated and a cannula (3) is used to flush the organ with heparinized saline solution in order to remove excess blood (a). The organ is then connected to a perfusion machine (b) and infused with autologous porcine blood for 6 hours. The perfusion machine is composed of an organ chamber (1), a blood reservoir (2), a centrifugal pump (3), a water bath (4), an oxygenator (5), a pressure sensor (6), a connector for injections and sample collection (7), and the main control panel (8). 
arterial temperature to reach $37^{\circ} \mathrm{C}$ ) while the oxygen supply was provided by an oxygenator at a rate of $2 \mathrm{l} / \mathrm{min}$. Perfusion was performed at a constant pressure of $80 \mathrm{Hg} / \mathrm{mm}$ for the duration of the experiment. Perfusion was maintained for $6 \mathrm{~h}$ and the oxygenated blood was recirculated from the reservoir via the perfusion circuit to the splenic artery (Fig. 1b). Epoprostenol sodium $(500 \mu \mathrm{g})$ was added to prevent vascular stricture due to ischemic-reperfusion injury (Gravante et al., 2009, 2012), thus increasing vascular dilatation to provide sufficient oxygen supply.

\section{Bacteria preparation and inoculation}

The S. pneumoniae strain D39 (NCTC 7466) was grown in tryptic soy broth (TSB) medium and frozen stock aliquots containing $6.5 \times 10^{7}$ colony forming units (CFU) were prepared in TSB $+10 \%$ glycerol. For each infection experiment, whole stock of pneumococci was thawed on ice and inoculated into the circuit $1 \mathrm{~h}$ after the start of perfusion.

Additional bacterial strains were assayed for survival in porcine blood to explore the scope of this assay. These were Enterococcus faecalis (ATCC 29212), Escherichia coli (ATCC 25922), Listeria monocytogenes (ScottA), Pseudomonas aeruginosa (ATCC 10115), Salmonella enteritidis (SE147), and Staphylococcus aureus (ATCC 43300). These strains were grown in brain heart infusion (BHI) broth, and frozen aliquots were prepared in $\mathrm{BHI}+10 \%$ glycerol. For growth assays, $1 \times 10^{6} \mathrm{CFU} / \mathrm{ml}$ of each bacterial strain were inoculated into $10 \mathrm{ml}$ of heparinized pig's blood and grown for $6 \mathrm{~h}$ at $37^{\circ} \mathrm{C}$. Samples were serially, ten-fold diluted and plated on BHI agar plates every hour to check the CFU numbers.

\section{Blood and serum analysis}

Arterial blood gas analysis was performed for each experiment immediately prior to bacterial inoculation and $2 \mathrm{~h}$ and $4 \mathrm{~h}$ postinfection. Several blood parameters were analyzed, including: $\mathrm{pH}$, hemoglobin, glucose, lactate, $\mathrm{Na}^{+}, \mathrm{Ca}^{2+}, \mathrm{K}^{+}, \mathrm{Cl}^{-}$to monitor the status of the organ and the possible development of acidosis or necrosis. Sera were collected every hour from both infected and uninfected control spleens. The sera were quantitatively tested for cytokines to measure the effect of the bacterial infection on immune regulation. In particular, interleukin-6 (IL-6) (R\&D, P6000B) and tumor necrosis factor- $\alpha$ (TNF- $\alpha)$ (R\&D, PTA00) concentrations were measured by solid phase sandwich ELISA. IL- 6 and TNF- $\alpha$ concentrations were calculated by comparing their dose response curves to the standard curves.

\section{Microscopy}

Biopsies of the perfused pig spleens were collected at $0 \mathrm{~min}$, $30 \mathrm{~min}, 2 \mathrm{~h}, 4 \mathrm{~h}$, and $5 \mathrm{~h}$ after inoculation of bacteria or PBS (negative controls). Samples were rapidly frozen in optimal cutting temperature (OCT) medium using dry ice and 2-methyl butane before sectioning using a cryostat (Leica CM1850UV). $10 \mu \mathrm{m}$ slices were sectioned and fixed with $4 \%$ paraformaldehyde on gelatin-coated slides. For confocal microscopy, samples were stained with several primary and secondary antibody combinations (see Tab. 1) to discern different cell types as previously described (Ercoli et al., 2018). Nuclei were stained with DAPI. The mouse spleen image was acquired from a naïve CD1 mouse spleen section. The mouse was part of a cohort used in a previous study (Ercoli et al., 2018). Briefly, the animal was euthanized according to the Animal (Scientific Procedures) Act 1986, spleen was sliced after freezing it in OCT and then stained as described above. The antibodies used were a rabbit anti-CD169 (Biorbyt orb385451, metallophilic macrophages) and a combination of rat anti-B220 (Biolegend 103201, B cells) and rat anti-CD3 (Abcam ab33429, t cells) antibodies. Imaging was performed with an Olympus FV1000 confocal laser scanning microscope with $20 \mathrm{X}$ and $60 \mathrm{X}$ objectives. Scanning images of whole spleen sections were performed with a fully motorized Nikon Eclipse Ti microscope equipped with a Plan Fluor 10X/0.3 objective and an Andor iXonEM+ EMCCD DU 885 camera, with the NIS-

Tab. 1: Antibodies and microscopy reagents

\begin{tabular}{|l|l|l|l|l|}
\hline Antibody & Specificity & Conjugated & Catalogue & Supplier \\
\hline Anti-porcine CD169 3B11/11 & CD169+ MФ & no & MCA2316GA & Biorad \\
\hline Anti-porcine CD163 2A10/11 & CD163+ MФ & no & MCA2311GA & Biorad \\
\hline Anti-human CD3 | CD3-12 & T cells & no & MCA1477A488 & Biorad \\
\hline Anti-porcine 6D10 & Neutrophils & no & MCA2599GA & Biorad \\
\hline Anti-pneumococcal type 2 capsule & Bacteria & no & 16745 & Statens Serum Institut \\
\hline Chicken anti-rabbit IgG (H+L) & Secondary Ab & Alexa Fluor ${ }^{\circledR} 488$ & A-21441 & Thermoscientific \\
\hline Goat anti-rat IgG (H+L) & Secondary Ab & Alexa Fluor ${ }^{\circledR} 568$ & A-11077 & Thermoscientific \\
\hline Chicken anti-rat IgG (H+L) & Secondary Ab & Alexa Fluor ${ }^{\circledR} 647$ & A-21472 & Thermoscientific \\
\hline Goat anti-mouse IgG (H+L) & Secondary Ab & Alexa Fluor ${ }^{\circledR} 568$ & A-11004 & Thermoscientific \\
\hline Antifade mountant with DAPI & DNA & DAPI & P36962 & Thermoscientific \\
\hline Phalloidin & Actin & Alexa Fluor ${ }^{\circledR} 647$ & A22287 & Thermoscientific \\
\hline
\end{tabular}


Elements software. Finally, samples were dried for 5 min and then stained with hematoxylin and eosin, as previously described (Fischer et al., 2008). All images were processed with Olympus Fluoview and ImageJ software (Schneider et al., 2012).

\section{Neutrophil quantification}

Neutrophils were detected by microscopy on tissue sections using a Ly-6G-specific primary antibody (Tab. 1). Perfused spleen sections at $0 \mathrm{~h}, 3 \mathrm{~h}$, and $5 \mathrm{~h}$ after infection were analyzed, an uninfected $5 \mathrm{~h}$ perfused spleen was also used as a negative control. Mean fluorescence intensity (MFI) was calculated using ImageJ software (Schneider et al., 2012). Neutrophils were counted in at least 15 fields from three independent biological replicates.

\section{Statistical analysis}

GraphPad Prism software version 6 was used to analyze all data. One-way ANOVA, with Tukey's post-hoc test, was used to compare neutrophil distribution in the different samples. Results were considered significant when $p$ values were $<0.05$. Error bars in all figures show the standard deviation (SD), unless otherwise stated.

\section{Results}

\subsection{Set-up of the normothermic porcine spleen perfusion model}

A spleen perfusion was set up on organs retrieved after slaughter. Therefore, in the final protocol several steps have been included to prevent coagulation, ischemic damage and necrosis. Porcine spleens were collected immediately after slaughter at the local abattoir. The organs were retrieved from animals bred exclusively for food production, thus the development of this model allowed replacement of protected animals. Following laparotomy, spleens were collected and the splenic artery cannulated while all the other arteries were ligated (Fig. 1a). Spleens were then flushed with 11 of saline solution containing heparin (5000 IU) to remove the blood trapped in the organ. In parallel, blood from the same pig was collected and treated with heparin to avoid coagulation. Both organ and blood were then transported to the hospital where the extracorporeal perfusion circuit was already prepared. The set-up included a circuit that is composed of a centrifugal pump, a heat exchanger, an oxygenator, and a reservoir for the blood (custom-made circuit, Medtronic) (Fig. 1b). All these components are connected to a perfusion machine (560 1.6 v Bio Console, Medtronic, 560 BCS1) that controls the blood pressure and constantly measures the flow rate (TX-50P Flow Transducer, Medtronic, 95185003). Before connection to the circuit, the organ was flushed with urokinase (10,000 IU) to dissolve residual blood clots and then again with saline solution. The circuit was filled with 11 of heparinized blood (25,000 IU) and the flow was activated. At this stage, the spleen was connected to the circuit through the cannula that had been ligated to the main artery and the blood started to perfuse the spleen.

This ex vivo perfusion technique was previously developed and refined in our lab using porcine livers and kidneys (Gravante

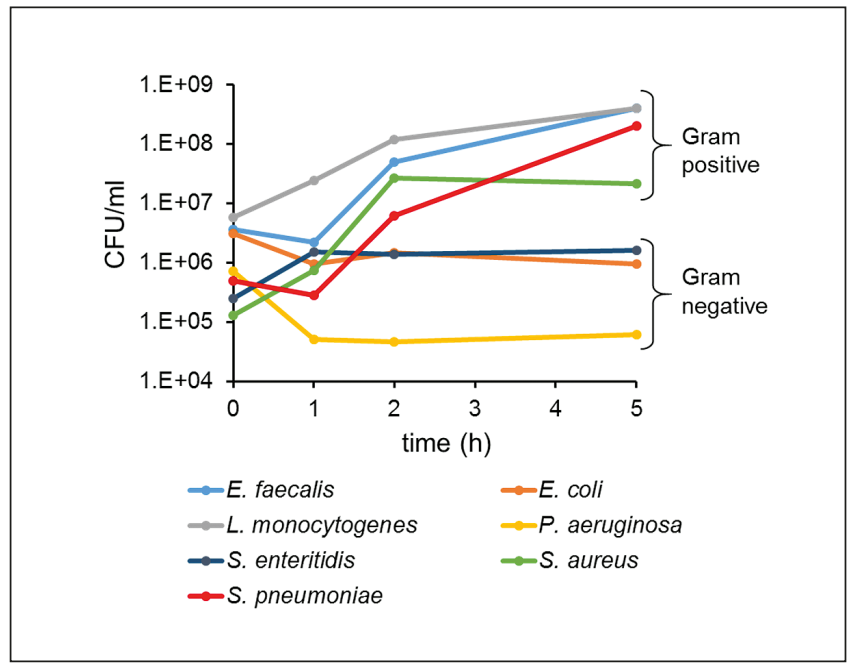

Fig. 2: Survival of different bacterial species in heparinized porcine blood

A panel of Enterococcus faecalis strain ATCC 29212, Escherichia coli strain ATCC 25922, Listeria monocytogenes strain ScottA, Pseudomonas aeruginosa strain ATCC 10115, Salmonella enteritidis strain SE147, and Staphylococcus aureus strain ATCC 43300 was tested for growth and survival in porcine blood. $1 \times 10^{6}$ CFU of each strain were incubated in whole heparinized pig's blood for 5 hours and samples were plated for CFU counting every hour (data show a representative experiment from three independent experiments). Gram-positive bacteria were better capable of survival and growth in porcine blood, while Gram-negative bacteria survived, but did not grow.

et al., 2012). Throughout these studies, numerous technical modifications have improved the circuit (particularly the cardio-vascular component), and - as a consequence - also the organ viability throughout the experimental time course (Chung et al., $2015,2012 b, 2013)$. Nevertheless, the organ stress response to the harvesting and reperfusion processes (Gravante et al., 2009), in addition to the lack of homeostatic organs (kidneys, lungs) (Gravante et al., 2010), prevented the physiologic environment within the circuit to be stable for periods of more than 6 hours. To avoid thrombosis and combinations of acid-base changes (Gravante et al., 2012) that can potentially affect the organ viability and function, an infusion saline bag containing Epoprostenol sodium $(500 \mu \mathrm{g})$ and heparin $(5,000 \mathrm{IU})$ was connected to the system with a flow of $20 \mathrm{ml} / \mathrm{h}$. Glucose $(5 \%)$ was infused at $5 \mathrm{ml} / \mathrm{h}$ to provide nutrients for the tissues. The established settings adapted for the porcine spleen allowed us to run the perfusion for 6 hours without experiencing major changes in the organ physiological parameters, which is a sufficient time to study the early stages of bacterial sepsis.

\subsection{The perfused spleen as a model of infection to study bacterial sepsis}

The porcine spleen perfusion model was tested as an alternative to the commonly used animal models to study the early stages of bacterial sepsis. To assess whether this model could be used 
A

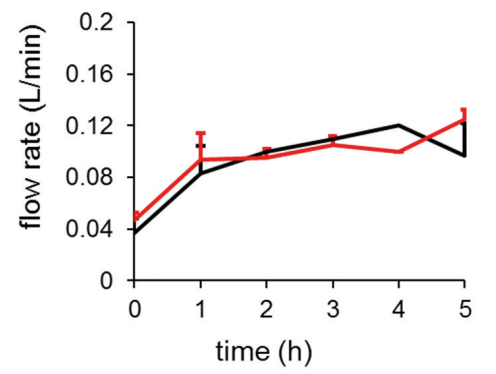

-uninfected

-infected
B
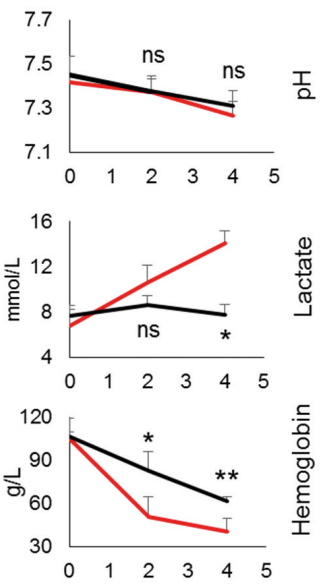

C
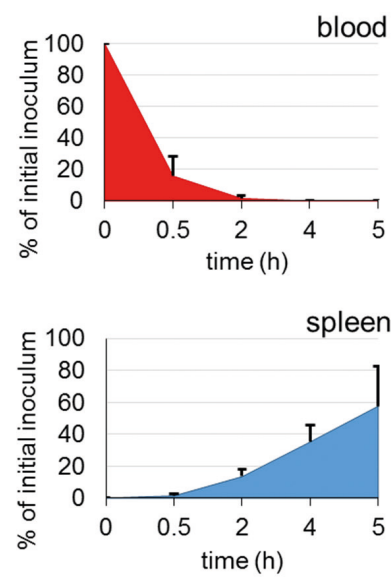

\section{Fig. 3: Measurement of perfused spleen blood parameters}

One hour after the start of perfusion, the spleen was injected with $6.5 \times 10^{7}$ CFU of $S$. pneumoniae $(n=4)$ or with PBS $(n=3)$. The perfusions were run for 5 hours after the injection and serial blood samples and spleen biopsies were collected at defined time-points. Blood pressure was kept constant at $80 \mathrm{mmHg}$ and the flow rate was measured during the perfusions as a marker of organ functionality. The flow rate observed both in the infected (red line) and in the uninfected (black line) samples was stable and within physiological parameters (A). Blood parameters were measured to exclude the occurrence of acidosis resulting from the formation of ischemic areas and necrosis. Values for $\mathrm{pH}$, lactate, and hemoglobin are reported for the infected (red line) and the uninfected (black line) blood samples. A t-test was used for statistical analysis ( ${ }^{*}, p<0.05$; ${ }^{*}, p<0.01$; ns, not significant) (B). CFU counts were performed on blood samples and serial biopsies in the infected spleens and showed that bacteria injected into the circuit were gradually trapped by the spleen and disappeared from the blood after 4 hours (C). After 2 hours, $20 \%$ of the original bacteria were shown to be trapped in the spleen and continued to increase over time, probably due to bacterial replication (D).

to study different bacterial species, a panel of bacterial strains was tested for growth and survival in porcine blood. The results revealed that Gram-negative bacteria could survive but not proliferate in porcine blood, while all Gram-positive bacteria were capable of survival and replication (Fig. 2). This finding is in line with the superior complement resistance of Gram-positive bacteria and highlighted their better suitability for use in this model. The Gram-positive bacterium Streptococcus pneumoniae was selected for further infection experiments, not only because it is often associated with sepsis in humans, but especially because of the crucial role that the spleen is reported to play in fighting systemic infections caused by this bacterium (Deniset et al., 2017; Hosea, 1983; Brown et al., 1981; Ercoli et al., 2018). To minimize bacterial contamination, the autologous blood used for the perfusion was pre-treated with antibiotics (10 $\mu \mathrm{g} / \mathrm{ml}$ colistin and $5 \mu \mathrm{g} / \mathrm{ml}$ nalidixic acid) that are not active against $S$. pneumoniae at these concentrations. One hour after the start of perfusion, organs were injected with $6.5 \times 10^{7} \mathrm{CFU}$ of $S$. pneumoniae $(\mathrm{n}=4)$ or with PBS as a negative control $(\mathrm{n}=3)$. Perfusions were run for 5 hours post-infection (6 hours in total). Serial blood and serum samples, and spleen biopsies were collected at defined time-points to analyze whether the organ was able to maintain its clearance capacity under these conditions, and if the effects of bacterial infection on the ex vivo spleen mirrored those observed in vivo using experimental animals.

\subsection{Assessment of organ functionality}

The functionality of splenic circulation was assessed by checking that the organ was bleeding only from the main splenic vein. Keeping the blood pressure constant at $80 \mathrm{mmHg}$, the flow rate rapidly increased and became stable between $0.1-0.15 \mathrm{l} / \mathrm{min}$ for the duration of the perfusion (Fig. 3a). These parameters provide a measurement of the resistance of the organ and were monitored for the remainder of the perfusion (Fig. 3a). An excessive increase in the flow rate denotes that the organ no longer provides physiological resistance and may start to enlarge, affecting the filtering functionality. Blood gas analysis was performed on blood samples collected at the beginning of the perfusion, after 2 hours and 4 hours. Glucose, lactate, $\mathrm{pH}, \mathrm{O}_{2}, \mathrm{CO}_{2}$, hemoglobin, and electrolyte concentrations (all key facets of functional organ physiology) were measured throughout the perfusion and infection procedures. These parameters were tracked over time to exclude the occurrence of acidosis resulting from the formation of ischemic areas and necrosis. The blood gas values, $\mathrm{pH}$ (Fig. $3 \mathrm{~b}$ ), and electrolyte levels of the uninfected group (Fig. S1 ${ }^{1}$ ) remained stable throughout the duration of the perfusion, thus

1 doi:10.14573/altex.1805131s 
A

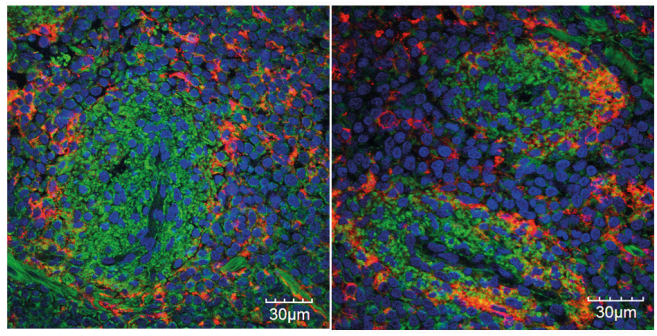

B

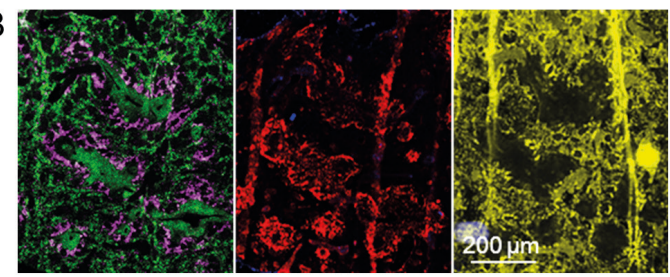

C
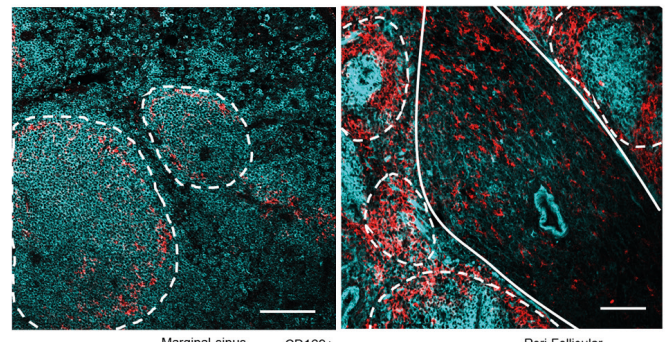

D
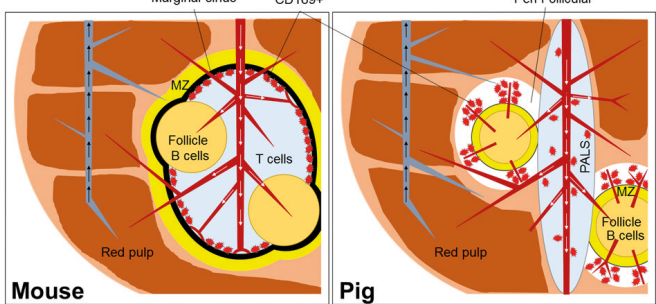

Fig. 4: Splenic architecture is maintained during perfusion

Immunohistochemistry and fluorescent microscopy were performed to assess the organ architecture and immune cell composition after perfusion and bacterial uptake. Microscopy on the perfused spleens $(n=7)$ was performed at three different time points $(0,2$ and 5 hours) and three different sections for each biopsy were analyzed. (A) Representative fluorescent microscopy images of uninfected spleen sections showed a preserved morphology and immune cell distribution at the beginning (left panel) and at the end (right panel) of the organ perfusion. (B) Subsequent sections of spleen perfused for 6 hours were stained with three antibody combinations to confirm the absence of changes in immune cell distribution. T cells are shown in green, neutrophils in magenta (left panel), CD169+ macrophages (indicating the peri-follicular area) in red (middle panel), and CD163+ macrophages (indicating the red pulp) in yellow (right panel). (C) Direct comparison of splenic architecture of mouse (left panel, archive picture) and pig (right panel). CD169+ cells are shown in red in both samples, while the light blue staining indicates the white pulp in the mouse spleen (combination of anti-B220 and anti-CD3 antibodies), and the follicles and endothelium in the pig spleen (phalloidin). (D) Schematic representation of the comparison of the splenic micro-architecture of the two species, showing the difference in follicle organization and in the CD169+ cell distribution.

confirming the absence of necrotic processes and acidosis. In the infected group, lactate and hemoglobin increased and decreased, respectively, thereby showing effects on the organ caused by infection (Fig. 3b). Of note, the $\mathrm{pH}$ levels remained within the physiological levels also in the infected spleen (Fig. 3b). We assessed the organ clearance capacity by measuring its ability to gradually trap the bacteria. CFU counts in blood samples demonstrated that the ex vivo organs were capable of complete blood bacterial clearance after 4 hours (Fig. 3c). Interestingly, in an inverse correlation, the number of bacteria in the spleen increased over time due to bacterial replication, in accordance with what we have recently reported for S. pneumoniae (Ercoli et al., 2018).

\subsection{The architecture of the porcine spleen after perfusion}

Conservation of splenic architecture after perfusion was assessed by microscopy. Confocal imaging on multiple uninfected spleen sections confirmed the absence of defects in the organ structure after perfusion. As shown in Figure 4a, we could not observe significant differences in the organ structure between the beginning (left panel, $\mathrm{t}=0$ ) and the end (right panel, $\mathrm{t}=6 \mathrm{~h}$ ) of the perfusion. To analyze spleen microarchitecture and char- acterize the immune cell distribution in the perfused organs, three adjacent $10 \mu \mathrm{m}$ sections (to ensure identical microarchitecture) were prepared from uninfected pig spleens following 6 hours of perfusion and were stained for peri-follicular macrophages (CD169+), red pulp macrophages, T cells, and neutrophils (Fig. 4b). The repertoires of primary and fluorescently labelled secondary antibodies used for fluorescent imaging are shown in Table 1. Staining of CD169+ macrophages allowed identification of the peri-follicular areas, distributed in ring structures across the section (Fig. 4b; red staining). Areas of red pulp could be identified by fluorescent imaging of red pulp macrophage populations (Fig. 4b; yellow stain), making up the vast majority of the spleen section. Finally, T cells could be identified in the peri-arteriolar sheets (PALS), whereas neutrophil populations were found in the red pulp (Fig. 4b; green and magenta staining, respectively). Overall, microscopy analysis showed a good preservation of the immune cell distribution and we could not observe any abnormalities in the follicle and PALS structure.

Since CD169+ macrophages have been recently indicated as crucial for pneumococcal splenic infection (Ercoli et al., 2018), we compared their distribution in mice and pigs (Fig. 4c). Spleen sections were stained to identify the localization of follicles 
A
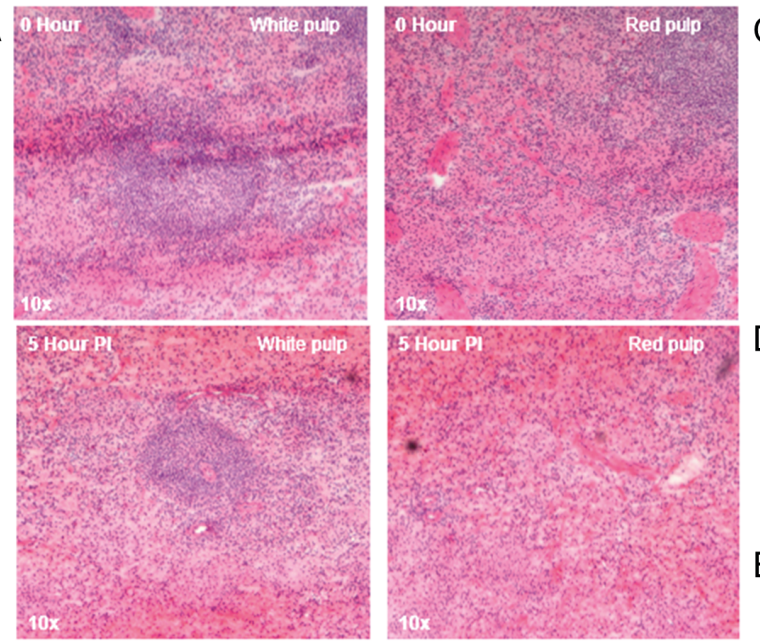

B

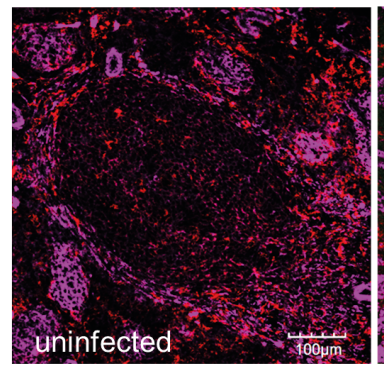

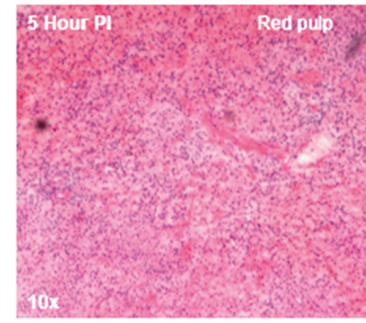

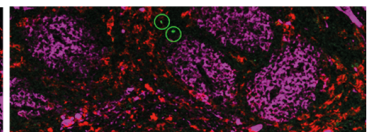

C
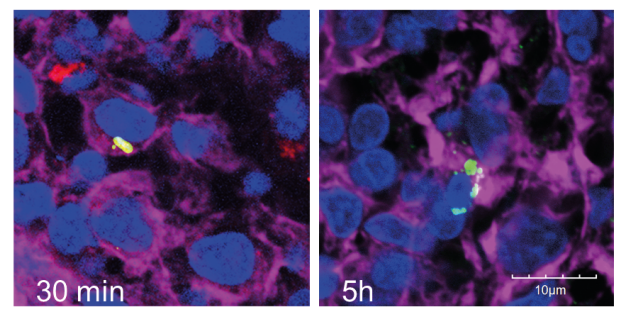

D

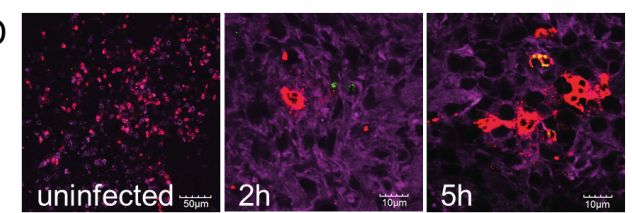

E
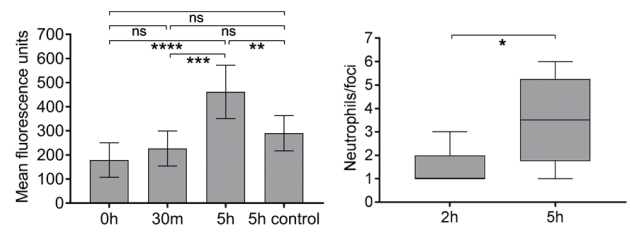

F
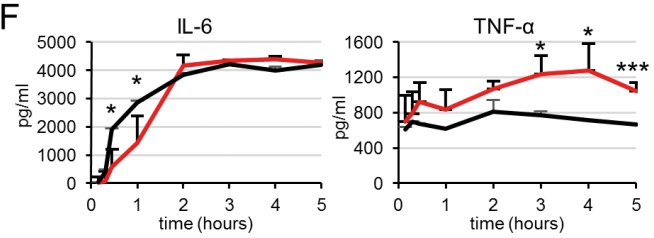

Fig. 5: Clusters of replicating pneumococci have been found in the perfused spleen

(A) Hematoxylin and eosin staining of infected spleen sections showed good preservation of the splenic tissue at all time points. The white pulp (left panel) and red pulp (right panel) show a clear demarcation at early stages of perfusion (top panels). At 5 hours, the red pulp/ white pulp demarcation is slightly less clear due to expansion of the marginal zone at the periphery of the white pulp (bottom panels).

(B) Confocal microscopy analysis of spleen sections showed the appearance of clusters of pneumococci (indicated by green circles) within the tissue after perfusion and infection, but no overall difference in the splenic architecture. (C) Single pneumococci (green) are trapped immediately after the infection by the perfused spleen (left panel). At later time points an increase in number of bacterial cells in each cluster was observed (right panel). Staining was performed using anti-type 2-capsule (green) and anti-pig CD169 primary antibodies (red), 647-conjugated phalloidin (magenta) and DAPI (blue). (D) Neutrophil localization and recruitment in the perfused spleens were analyzed by microscopy. 5 hours perfused uninfected (left panel), 2 hours infected (middle panel) and 5 hours infected (right panel) spleen sections were stained. In the red pulp (magenta), neutrophils (red), which are in the proximity of bacterial clusters (green), increase in number over time. (E) A measurement of the mean fluorescence intensity (MFI) of neutrophils in these tissue sections at different time points showed an increase only at the late stage of splenic infection. Numbers of neutrophils in proximity to bacterial clusters were also counted, and showed an increase over time, likely due to chemotactic recruitment. 15 fields from 3 independent samples were analyzed for each time point. Error bars indicate standard deviation. All the microscopy on the perfused spleens $(n=7)$ was performed at three different time points $(0,2$, and 5 hours $)$ and three different sections for each biopsy were analysed, representative images are shown. (F) IL- 6 and TNF- $\alpha$ levels were measured by ELISA assay in the sera collected from the infected (red lines, $n=4$ ) and uninfected (black lines, $n=3$ ) blood during the perfusion experiment. Lines indicate the medians, error bars the standard deviation. One-way ANOVA with Tukey's post-hoc test was used for statistical analysis ( ${ }^{*}, p<0.05 ;{ }^{* *}, p<0.01 ;{ }^{* * *}, p<0.001 ;{ }^{* * * *}, p<0.0001 ;$ ns, not significant).

(light blue) and CD169+ cells (red). As expected, we observed the CD169+ cells to only localize at the edge of the white pulp in mice. In pigs, the CD169+ macrophages were mainly localized in the peri-follicular area, with some found in the PALS, in close analogy to what is reported for humans (Mebius and Kraal, 2005). In Figure 4d, a model of the CD169+ macrophage distri- bution in mice and pigs is reported. There are clear differences in the structure of the organ and in the distribution of the CD169+ cells between the two species, suggesting that at least for pneumococcal infection - which is caused by a human specific pathogen - the use of a model that is more similar to humans can help in the translation of future findings. 


\subsection{Early stages of bacterial sepsis in the spleen perfusion model}

Spleen sections were analyzed after infection to investigate the presence of any tissue damage caused by the infection. To determine the level of preservation of the organ during perfusion, a number of different immunohistochemistry and histological stainings were performed on $10 \mu \mathrm{m}$ sections from both uninfected and infected spleen biopsies. Hematoxylin and eosin staining was used to investigate the viability of the tissue across the infection time course, and as a validation of this model for histology studies. We observed a good preservation of the splenic tissue after perfusion in the control uninfected sample (Fig. S2 ${ }^{1}$ ) and also in the infected spleen both at the beginning ( 0 hours) and at the end (5 hours) of the infection (Fig. 5a). The red pulp and white pulp were clearly demarcated in control spleen and at the early stages of infection. At 5 hours, the red/white pulp demarcation was slightly less clear due to expansion of the marginal zone at the periphery of the white pulp. This expansion is due to an increased number of cells with the histological features of macrophages.

Confocal microscopy also confirmed that the microarchitecture of the organ was preserved 5 hours after infection (Fig. $5 \mathrm{~b}$ ). A timecourse analysis was performed on spleen biopsies collected from each experiment, looking at bacterial numbers and distribution. As seen in Figure 3c, the bacterial number increased in the spleen even when the blood was fully cleared. This suggested bacterial replication taking place within the perfused spleen, and confocal microscopy confirmed the formation of clusters of bacteria within splenic cells at later time-points (Fig. 5b). The number of bacteria in each cluster increased over time and was maximal at the last time-point (Fig. 5c). As we observed formation of foci of infection in the spleen, we tested whether non-resident immune cell subtypes were recruited to the infection site. Neutrophils, in particular, were observed to increase in number during the infection and to co-localize with the bacteria in the red pulp (Fig. 5d,e). The average number of neutrophils surrounding a bacterial cluster also increased with time (Fig. 5e), showing that the organ was still capable of signaling for alternative innate clearance mechanisms to the phagocytosis mediated by resident macrophages to fight the infection. In the uninfected control perfusion, the neutrophil number was comparable to that of the early time points (Fig. 5e).

Macrophage release of IL- 6 and TNF- $\alpha$ in the blood as a response to bacterial infection was also tested (Fig. 5e). Although the slaughtering process and the non-sterile collection of the blood may initiate cytokine release before bacterial challenge, as has been reported for TNF- $\alpha$ (Liu et al., 2010), we found significantly higher levels of this cytokine in the infected sample than in the uninfected sample. Despite having a lower starting concentration, IL-6 increased at the same rate in both uninfected and infected samples, and the levels reached were comparable to what was observed in previous ex vivo organ perfusion studies (Gravante et al., 2009; Chung et al., 2012a).

\section{Discussion}

A porcine spleen perfusion model of infection was established using organs retrieved from routine slaughter for food production.
The development of this technique involved the refinement of three different processes: organ collection, perfusion, and bacterial infection. Special care was taken during organ collection, especially in removal of all blood trapped in the spleen after slaughter. Failure in this process could result in the blockage of blood circulation during perfusion and formation of ischemic areas, which would hamper experimental studies. Extensive washes with anticoagulant solution and thrombolytic agents were applied to prevent blood vessel damage. Moreover, a final confirmation of the absence of obstacles to blood circulation was provided by the measurement of blood flow and pressure during the perfusion process. The perfusion process was monitored constantly to assess organ viability and functionality. Blood gas analysis and immunohistochemistry were performed to exclude major changes in the functionality and microarchitecture of the organ as a result of the perfusion process. Whilst less than optimal blood-gas parameters were obtained at the start of the infection - likely a result of the damage incurred during slaughter - physiologically relevant conditions were still achieved throughout the experiment. Regarding bacterial infection, the major bacterial human-specific pathogen S. pneumoniae was tested with respect to dose and time of infection. Studies of the effect of pneumococcal infection in the perfused spleens were investigated, focusing on splenic clearance, cytokine release (starting cytokines levels are also affected by the slaughtering process), neutrophil recruitment and bacterial survival. We showed that a number of features of our model mimic those of data shown in mice and humans: rapid clearance of pneumococci from the blood, release of tumor necrosis factor following infection, neutrophil recruitment across the infection site, and, more recently, intracellular replication of pneumococci within splenic macrophages (Ercoli et al., 2018).

Since 2005 our group has used an ex vivo perfused porcine organ model to study organ physiology and investigate the implications of using these models with respect to human physiology (Chung et al., 2013; Gravante et al., 2009, 2010, 2012). Most of the biochemical, immunologic, and pathological changes observed in this model are very similar to those observed in animal research, bridging the gap between in vitro and in vivo studies (Daniel et al., 2018). This confirms that our model is a reliable and inexpensive method for the study of organ physiology and, as shown with this study, also its response to systemic infection (Gravante et al., 2009, 2010, 2012). Importantly, an ex vivo spleen circuit is also disconnected from physiological extrinsic regulatory control systems (neurological, hormonal, or chemical), which are present in complex organisms, and allows for a detailed focused evaluation of the organ's intrinsic response to infectious stimuli. The use of perfused porcine spleens to study bacterial sepsis is not only advantageous because of the replacement of experimental animals, but it also provides a model that is very similar to humans that could both help in the translation of rodent studies and to predict the efficacy of antimicrobial therapies. In our study, we have focused on the optimization of this model as a tool to study host-pathogen interactions. The possibility to perform multiple sampling, time-course studies, and live imaging on the same perfused organ opens the road to investigate the fine dynamics of the strategies that both bacteria and host employ during infection. 
This innovative system is flexible (different organs and pathogens can be tested), reduces the number of required experiments (as multiple and/or serial sampling can be performed on the same organ), and improves the translatability of the data generated (since porcine organs are much more similar to those of humans than are those of rodents). For these reasons, our model addresses the unmet need for an ex vivo model in the fields of infection and anti-infective drug discovery and will be particularly important for the study of the initial phases of invasive diseases. A future application of the model could be testing the efficacy of antimicrobial compounds with particular emphasis on intracellular pathogen eradication. New refinements of the organ collection will be tested in order to prolong the perfusion time up to 12 hours. Reduction of ischemic damage will be the key point, mainly solvable by reducing the collection time (surgery) and by improving the flushing technique (using thrombolytic agents). In this way, the bactericidal activity of new molecules could be assessed in a system resembling the early stages of bacteremia. Organ perfusion infection models have been developed in the past, both with rodent and human organs. Mouse liver and rat intestine have been tested respectively with Candida albicans and Salmonella enteritidis to investigate host-pathogen interactions (Schwocho and Moon, 1981; Boyle et al., 2016), while human spleen was infected with the parasite Plasmodium falciparum to test organ clearance and processing functionality in pathophysiological conditions (Buffet et al., 2006). The rodent organ perfusions did not overcome the limitations of studying infectious diseases in a poorly relevant model, and the human system is limited by human sample availability. Importantly, the key advantage of our work is the replacement of animals used for research with abattoir sourced organs.

Due to multiple samplings in our model, the data collected from one single infected pig organ (in our experimental series) correspond to data obtained using 48 mice. Since January 2015 more than 1200 articles have been published using mice to study organ-specific infections caused by Streptococcus pneumoniae, Staphylococcus aureus, Listeria monocytogenes, uropathogenic (UPEC) Escherichia coli, and Salmonella enterica. An estimate based on the average reported number of mice used in $5 \%$ of these papers brings the total number of mice to 71,600 for these studies. Obviously, this estimate is conservative as it only accounts for animals reported in publications, thus the real number of experimental animals in use is strongly underestimated. Moreover, this calculation only refers to mice and does not take into consideration other animal species that might be used for $e x$ vivo perfusion models of infection. Piglet infection models are the most direct comparison to our model. Since January 2015, about 90 articles have been published reporting the use of more than 2900 piglets to study bacterial infections.

In conclusion, we report the development of an ex vivo porcine spleen perfusion model sourced from pigs slaughtered for food production, optimized for the study of bacterial infection. We show that organ function is maintained for 6 hours - sufficient time to investigate the early events preceding sepsis. This model mimics many hallmarks of bacterial disease, including rapid clearance of bacteria from the blood and increases in pro-in- flammatory cytokine production following infection. We also demonstrate the utility of this model for histological studies. Most importantly, this model provides an avenue for translation of data obtained in rodents to immunologically distinct humans and reduces the need for use of experimental animals.

\section{References}

Boxall, T. (1969). A physiological study of the isolated spleen. Ann R Coll Sur Engl 45, 277-293.

Boyle, E. C., Dombrowsky, H., Sarau, J. et al. (2016). Ex vivo perfusion of the isolated rat small intestine as a novel model of salmonella enteritis. Am J Physiol Gastrointest Liver Physiol 310, G55-63. doi:10.1152/ajpgi.00444.2014

Brogaard, L., Heegaard, P. M. H., Larsen, L. E. et al. (2016). Late regulation of immune genes and microRNAS in circulating leukocytes in a pig model of influenza A (H1N2) infection. Sci Rep 6, 21812. doi:10.1038/srep21812

Brown, E. J., Hosea, S. W. and Frank, M. M. (1981). The role of the spleen in experimental pneumococcal bacteremia. $J$ Clin Invest 67, 975-982. doi:10.1172/JCI110148

Buffet, P. A., Milon, G., Brousse, V. et al. (2006). Ex vivo perfusion of human spleens maintains clearing and processing functions. Blood 107, 3745-3752. doi:10.1182/blood-200510-4094

Butler, A. J., Rees, M. A., Wight, D. G. et al. (2002). Successful extracorporeal porcine liver perfusion for $72 \mathrm{hr}$. Transplantation 73, 1212-1218. doi:10.1097/00007890-200204270-00005

Chung, W. Y., Gravante, G., Al-Leswas, D. et al. (2012a). Addition of a kidney to the normothermic ex vivo perfused porcine liver model does not increase cytokine response. J Artif Organs 15, 290-294. doi:10.1007/s10047-012-0641-9

Chung, W. Y., Gravante, G., Al-Leswas, D. et al. (2012b). Addition of a kidney to the normothermic ex vivo perfused porcine liver model does not increase cytokine response. J Artif Organs 15, 290-294. doi:10.1007/s10047-012-0641-9

Chung, W. Y., Gravante, G., Al-Leswas, D. et al. (2013). The development of a multiorgan ex vivo perfused model: Results with the porcine liver-kidney circuit over 24 hours. Artif Organs 37, 457-466. doi:10.1111/aor.12003

Chung, W. Y., Gravante, G., Eltweri, A. et al. (2015). The "kidney-liver" multiorgan ex vivo perfused model improves the circuit's biochemical milieu during perfusion compared to the "liver-kidney" counterpart. J Artif Organs 18, 151-161. doi:10.1007/s10047-014-0813-x

Daniel, C. R., Labens, R., Argyle, D. and Licka, T. F. (2018). Extracorporeal perfusion of isolated organs of large animals Bridging the gap between in vitro and in vivo studies. ALTEX 35, 77-98. doi:10.14573/altex.1611291

Dawson, H. (2011). A comparative assessment of the pig, mouse and human genomes. In The Minipig in Biomedical Research. CRC Press. https://bit.ly/2PP1bnW

Dawson, H. D., Loveland, J. E., Pascal, G. et al. (2013). Structural and functional annotation of the porcine immunome. BMC Genomics 14, 332. doi:10.1186/1471-2164-14-332

Deniset, J. F., Surewaard, B. G., Lee, W. Y. and Kubes, P. (2017). Splenic Ly $6 \mathrm{G}^{\text {high }}$ mature and Ly6G ${ }^{\text {int }}$ immature neutrophils 
contribute to eradication of S. pneumoniae. J Exp Med 214, 1333-1350. doi:10.1084/jem.20161621

Ercoli, G., Fernandes, V. E., Chung, W. Y. et al. (2018). Intracellular replication of Streptococcus pneumoniae inside splenic macrophages serves as a reservoir for septicaemia. Nat Microbiol 3, 600-610. doi:10.1038/s41564-018-0147-1

EC (2009). Council regulation (EC) no 1099/2009 of 24 September 2009 on the protection of animals at the time of killing. http://data.europa.eu/eli/reg/2009/1099/oj

EU (2010). Directive 2010/63/EU of the European Parliament and of the Council of 22 September 2010 on the protection of animals used for scientific purposes. http://eur-lex.europa.eu/ eli/dir/2010/63/oj

Fink, M. P. (2014). Animal models of sepsis. Virulence 5, $143-$ 153. doi:10.4161/viru. 26083

Fischer, A. H., Jacobson, K. A., Rose, J. and Zeller, R. (2008). Hematoxylin and eosin staining of tissue and cell sections. CSH Protoc 2008, pdb.prot4986. doi:10.1101/pdb.prot4986

Fleetwood, G., Chlebus, M., Coenen, J. et al. (2015). Making progress and gaining momentum in global 3Rs efforts: How the European pharmaceutical industry is contributing. $J \mathrm{Am}$ Assoc Lab Anim Sci 54, 192-197.

Gravante, G., Ong, S. L., Metcalfe, M. S. et al. (2009). Cytokine response to ischemia/reperfusion injury in an ex vivo perfused porcine liver model. Transplant Proc 41, 1107-1112. doi:10.1016/j.transproceed.2009.02.054

Gravante, G., Ong, S. L., Metcalfe, M. S. et al. (2010). Cytokine response of electrolytic ablation in an ex vivo perfused liver model. ANZ J Surg 80, 537-541. doi:10.1111/j.14452197.2010.05380.x

Gravante, G., Ong, S. L., Metcalfe, M. S. et al. (2012). Changes in acid-base balance during electrolytic ablation in an ex vivo perfused liver model. Am J Surg 204, 666-670. doi:10.1016/j. amjsurg.2009.12.019

Hein, W. R. and Griebel, P. J. (2003). A road less travelled: Large animal models in immunological research. Nat Rev Immunol 3, 79-84. doi:10.1038/nri977

Horan, M. and Colebatch, J. H. (1962). Relation between splenectomy and subsequent infection. A clinical study. Arch Dis Child 37, 398-414. doi:10.1136/adc.37.194.398

Hosea, S. W. (1983). Role of the spleen in pneumococcal infection. Lymphology 16, 115-120.

Imber, C. J., St Peter, S. D., de Cenarruzabeitia, I. L. et al. (2002a). Optimisation of bile production during normothermic preservation of porcine livers. Am J Transplant 2, 593-599. doi:10.1034/j.1600-6143.2002.20703.x

Imber, C. J., St Peter, S. D., Lopez de Cenarruzabeitia, I. et al. (2002b). Advantages of normothermic perfusion over cold storage in liver preservation. Transplantation 73, 701-709. doi:10.1097/00007890-200203150-00008

Liu, Y., Shi, W., Zhou, E. et al. (2010). Dynamic changes in in- flammatory cytokines in pigs infected with highly pathogenic porcine reproductive and respiratory syndrome virus. Clin Vaccine Immunol 17, 1439-1445. doi:10.1128/CVI.00517-09

Mak, I. W. Y., Evaniew, N. and Ghert, M. (2014). Lost in translation: Animal models and clinical trials in cancer treatment. $\mathrm{Am}$ $J$ Transl Res 6, 114-118.

Mebius, R. E. and Kraal, G. (2005). Structure and function of the spleen. Nat Rev Immunol 5, 606. doi:10.1038/nri1669

Mestas, J. and Hughes, C. C. (2004). Of mice and not men: Differences between mouse and human immunology. J Immunol 172, 2731-2738. doi:10.4049/jimmunol.172.5.2731

Meurens, F., Summerfield, A., Nauwynck, H. et al. (2012). The pig: A model for human infectious diseases. Trends Microbiol 20, 50-57. doi:10.1016/j.tim.2011.11.002

Mitka, M. (2011). Drug for severe sepsis is withdrawn from market, fails to reduce mortality. JAMA 306, 2439-2440. doi:10.1001/jama.2011.1755

Prescott, M. J. and Lidster, K. (2017). Improving quality of science through better animal welfare: The NC3Rs strategy. Lab Anim (NY) 46, 152-156. doi:10.1038/laban.1217

Schneider, C. A., Rasband, W. S. and Eliceiri, K. W. (2012). NIH Image to ImageJ: 25 years of image analysis. Nat Methods 9 , 671-675. doi:10.1038/nmeth.2089

Schwocho, L. R. and Moon, R. J. (1981). Clearance and killing of Candida albicans in the perfused mouse liver. Mycopathologia 76, 175-183. doi:10.1007/BF00437198

Seok, J., Warren, H. S., Cuenca, A. G. et al. (2013). Genomic responses in mouse models poorly mimic human inflammatory diseases. Proc Natl Acad Sci U S A 110, 3507-3512. doi:10.1073/pnas. 1222878110

Shinefield, H. R., Steinberg, C. R. and Kaye, D. (1966). Effect of splenectomy on the susceptibility of mice inoculated with Diplococcus pneumoniae. J Exp Med 123, 777-794. doi:10.1084/ jem.123.5.777

Steiniger, B. S. (2015). Human spleen microanatomy: Why mice do not suffice. Immunology 145, 334-346. doi:10.1111/ imm. 12469

Theilacker, C., Ludewig, K., Serr, A. et al. (2016). Overwhelming postsplenectomy infection: A prospective multicenter cohort study. Clin Infect Dis 62, 871-878. doi:10.1093/cid/civ1195

\section{Conflict of interest}

The authors declare no conflicts of interest.

\section{Acknowledgements}

The authors would like to thank Irene Vacca for reviewing the manuscript, the staff of Joseph Morris Butchers Ltd, Michael F. Wood Butchers and the staff of Leicester Preclinical Research Facility for support. 